McGill-91/24

July 1991

\title{
THE DAMPING OF ENERGETIC GLUONS AND QUARKS IN HIGH-TEMPERATURE QCD
}

\author{
C.P. Burgess and A.L. Marini* \\ Physics Department, McGill University, 3600 University St. \\ Montréal, Québec, Canada, H3A 2 T8.
}

\begin{abstract}
When a gluon or a quark is sent through the hot QCD plasma it can be absorbed into the ambient heat bath and so can acquire an effective lifetime. At high temperatures and for weak couplings the inverse lifetime, or damping rate, for energetic quarks and transverse gluons, (those whose momenta satisfy $|\mathbf{p}| \gg g T)$ is given by $\gamma(\mathbf{p})=c g^{2} \log \left(\frac{1}{g}\right) T+$ $O\left(g^{2} T\right)$. We show that very simple arguments suffice both to fix the numerical coefficient, $c$, in this expression and to show that the $O\left(g^{2} T\right)$ contribution is incalculable in perturbation theory without further assumptions. For QCD with $N_{c}$ colours we find (expressed in terms of the casimir invariants $C_{a}=N_{c}$ and $\left.C_{f}=\left(N_{c}^{2}-1\right) /\left(2 N_{c}\right)\right): c_{g}=+\frac{C_{a}}{4 \pi}$ for gluons and $c_{q}=+\frac{C_{f}}{4 \pi}$ for quarks. These numbers agree with the more detailed calculations of Pisarski et.al. but disagree with those of Lebedev and Smilga. The simplicity of the calculation also permits a direct verification of the gauge-invariance and physical sign of the result.
\end{abstract}

* internet: cliff@physics.mcgill.ca and alex@physics.mcgill.ca bitnet: py30@mcgilla 
The behaviour of nonabelian gauge theories at finite temperature is of theoretical interest due to the surprisingly rich structure they exhibit even within the perturbative regime of weak coupling and high temperatures. They may also have phenomenological applications to the interpretation of the collisions of heavy nuclei at high energies.

In recent years much attention has been directed towards understanding the properties that gluons and quarks acquire as they propagate through a quark-gluon plasma. ${ }^{1}$ This has been at least partially due to the sometimes contradictory and confusing results of the earliest one-loop calculations. In these calculations the gluon damping constant, $\gamma_{g}(\mathbf{p})$, was found in the static $(|\mathbf{p}| \rightarrow 0)$ limit to depend on the gauge on which it was computed and, for some gauges, to be negative. If taken seriously such a negative damping constant would indicate an instability of the thermal state towards gluon emission. The source of the confusion with these calculations ${ }^{2}$ is that they neglect higher-loop contributions that are of the same order in the gauge coupling constant, $g$, as are the terms that are kept. This is because in perturbation theory (in three space dimensions) at finite temperatures successive terms in the loop expansion need not be suppressed relative to fewer loops by additional factors of the gauge coupling.

The failure of the loop expansion arises at finite temperatures because of the occurence of severe infrared divergences. These infrared divergences are more troublesome than they are at zero temperature due to the singular behaviour of the Bose-Einstein distribution function at low energies: $n(E)=\left(e^{E / T}-1\right)^{-1} \approx \frac{T}{E}+\cdots$. This behaviour causes quantities to blow up like a power of the infrared cutoff rather than simply logarithmically as they do at zero temperature. ${ }^{3}$ Indeed, a simple power-counting argument shows that for QCD at temperature $T$ a generic $\ell$-loop graph can contribute an amount proportional to $\left(g^{2} T / \lambda\right)^{\ell}$ relative to the tree-level result. $\lambda$ in this expression is an infrared cutoff. Higher loops clearly need not be suppressed once $\lambda$ is as low as $g^{2} T$.

In fact the loop expansion already fails even for an infrared cutoff as large as $\lambda \approx g T$ since a subclass of diagrams can be even more infrared singular than is indicated by the generic power-counting argument. The dangerous graphs are those such as the 'ring' graphs within which multiple self-energy insertions are made along a single internal line. ${ }^{4}$ In reference [5] Braaten and Pisarski argue that these last contributions may be resummed by 
dressing all 'soft' lines - those carrying momenta less than or of order $g T$ - by the calculable contributions of 'hard thermal loops'. In ref. [2] they then compute the implications for the particular case of the damping rate for static gluons, arguing that the failure of perturbation theory is in this case completely cured by such a resummation. This last conclusion is, however, disputed by Lebedev and Smilga, ${ }^{6}$ who argue that other contributions beyond the hard thermal loops can contribute equally large effects to the static gluon damping constant.

The purpose of this letter is to point out that for at least some physical quantities the dominant part of the result for small $g$ can be identified quite simply without making use of the complete resummation formalism. We take by way of illustration the damping constant, $\gamma(\mathbf{p})$, for transverse gluons (and for quarks) but evaluated for momenta $|\mathbf{p}| \gg g T$ rather than in the static limit. For these momenta the dominant contribution for small coupling to $\gamma(\mathbf{p})$ is momentum independent and has the form $c g^{2} \log \left(\frac{1}{g}\right) T+b g^{2} T+\cdots$. We show that the coefficient $c$ can be computed by a simple, analytic calculation that only involves wavelengths that are within the perturbative regime: $\lambda \gg g^{2} T$. The same is not true for the coefficient $b$ which can receive contributions for $\lambda \approx g^{2} T$. We can in this instance therefore explicitly verify that $\gamma(\mathbf{p})$ is indeed gauge-independent and positive.

Our results, given in and immediately following eq. (12), may be compared to more detailed calculations. For fermions they agree with those of ref. [7] but disagree by a factor of 3 with the real-time calculations of ref. [6]. For transverse gluons we agree with the result of an as-yet-unpublished version of a full resummation calculation, ${ }^{8}$ but do not agree with the real-time estimate of ref. [6].

We now turn to a description of our calculation. We focus first on the purely gluonic theory since this is the case for which there is the most simplification over a full calculation.

The dispersion relation relating the energy and momentum for a relativistic transverse gluon traversing the plasma may be determined by the position, $E(\mathbf{p})=\omega(\mathbf{p})-i \gamma(\mathbf{p})$, in the complex energy plane of the zero of the transverse part of the inverse gluon propagator. Using the usual one-parameter family of covariant gauges for which the bare inverse Feynman propagator is:

$$
\left(G_{\text {bare }}^{-1}\right)_{\mu \nu}(p)=p^{2} \eta_{\mu \nu}+\left(\xi^{-1}-1\right) p_{\mu} p_{\nu}
$$


and the full thermal inverse propagator is:

$$
\left(G_{\text {full }}^{-1}\right)_{\mu \nu}(p)=\left(G_{\text {bare }}^{-1}\right)_{\mu \nu}(p)+\Pi_{\mu \nu}(E, \mathbf{p})
$$

the dispersion relation for transverse gluons becomes:

$$
E^{2}(\mathbf{p})-\mathbf{p}^{2}=\frac{1}{2}\left[\Pi_{i}^{i}-\frac{p^{i} p^{j} \Pi_{i j}}{\mathbf{p}^{2}}\right]
$$

Here $i$ and $j$ are to be summed over the three spatial directions, $i, j=1,2,3$, in the plasma rest frame. Our task is to compute the leading contributions to $\Pi_{\mu \nu}(E, \mathbf{p})$ and to, in particular, identify the origin of the $g^{2} \log g$ terms.

In order to sort out the size of the various contributions to $\Pi_{\mu \nu}$ it is useful to divide the loop integrations according to whether or not they involve only energies and momenta that are greater than some infrared cutoff, $\lambda$. That is:

$$
\Pi_{\mu \nu}(E, \mathbf{p})=\Pi_{\mu \nu}^{\mathrm{soft}}(E, \mathbf{p}, \lambda)+\Pi_{\mu \nu}^{\mathrm{hard}}(E, \mathbf{p}, \lambda)
$$

in which all loop momenta in $\Pi_{\mu \nu}^{\text {hard }}(E, \mathbf{p}, \lambda)$ are cut off in the infrared at $\lambda$. Provided that $\lambda$ is chosen sufficiently high $\Pi_{\mu \nu}^{\text {hard }}$ is calculable within the loop expansion. $\Pi_{\mu \nu}^{\text {soft }}$ is not computable in this way and must be obtained by other means. An important issue to be addressed is how much any desired quantity depends on the largely undetermined $\Pi_{\mu \nu}^{\text {soft }}$.

If $\lambda$ is chosen much greater than $g T$ then the lowest-order contribution to $\Pi_{\mu \nu}^{\text {hard }}$ arises at $O\left(g^{2}\right)$ due to one-loop graphs. There are four such one-loop graphs: one tadpole and one vacuum-polarization graph having either an internal gluon, quark or ghost loop. For example, using the Matsubara imaginary-time technique, ${ }^{9}$ the gluon vacuum-polarization graph contributes an amount:

$$
\begin{gathered}
\Pi_{\mu \nu}^{\mathrm{hard}}(E, \mathbf{p}, \lambda)=\frac{g^{2} C_{a} T}{2} \sum_{n=-\infty}^{\infty} \int_{\mathbf{q}^{2}>\lambda^{2}} \frac{d^{3} \mathbf{q}}{(2 \pi)^{4}} V_{\mu \lambda \rho}(p,-p-q, q) G^{\lambda \kappa}(p+q) \\
\times G^{\rho \sigma}(q) V_{\nu \sigma \kappa}(-p,-q, p+q) .
\end{gathered}
$$

Here the gluon propagator is the inverse of eq. (1) and the three-point gluon vertex is given by the zero-temperature expression: $V_{\mu \lambda \rho}(p, q, k)=(p-q)_{\rho} \eta_{\mu \lambda}+(q-k)_{\mu} \eta_{\rho \lambda}+(k-p)_{\lambda} \eta_{\mu \rho}$. The quadratic invariant in the adjoint representation is $C_{a}=N_{c}(=3)$ for the gauge group 
$S U\left(N_{c}\right)$. We work in Euclidean signature and the time component of every four-vector is an integer times $2 \pi T$. The summation is over the integer corresponding to the loop momentum, $q$. The lower limit on the momentum integration is meant as a reminder of the infrared cutoff.

If the infrared cutoff should instead be chosen to satisfy $g^{2} T \ll \lambda \ll g T$ then higherloop graphs can contribute to the same order in $g$ as do the one-loop graphs in which the loop momenta are of order $g T$. After resumming these higher-loop contributions the leading result for $\Pi_{\mu \nu}^{\text {hard }}$ is given by the same one-loop graphs as before, with the proviso $^{5}$ that each propagator (or vertex) is to be replaced with an 'effective' resummed propagator (or vertex): $G_{\mu \nu} \rightarrow G_{\mu \nu}^{*}$ and $V_{\mu \lambda \rho} \rightarrow V_{\mu \lambda \rho}^{*}$. Each of these resummed quantities agrees (up to higher powers of $g$ ) with its bare counterpart unless all of the momenta entering the line (or vertex) in question are themselves $O(g T)$. For such soft momenta, however, the resummed items are relatively complicated functions of the ratios of the energies and momenta that flow through that part of the graph. The main technical difficulty with working with the effective propagators and vertices lies in manipulating this more complicated form.

Some of this extra complication simplifies if the external momentum is itself chosen to be much larger than $g T$, as is the case for when the dispersion relation, $E(\mathbf{p})$, is evaluated for hard momenta such as $|\mathbf{p}| \approx T$. The reason for this simplification is that since the resummed and bare quantities agree if any of the relevant momenta are hard, $|\mathbf{p}| \gg g T$, it is only necessary to work with the resummed versions if all of the momenta passing through a particular line (or vertex) are soft. For hard external momenta many of the internal lines and vertices must also carry hard momenta and so may be represented by the usual bare Feynman rules. For example, in the vacuum-polarization graphs both of the vertices and one propagator must necessarily carry hard momenta if the external momentum is itself large in comparison to $g T$. This leaves at most a single internal propagator to be dressed.

Now comes the main point: the dominant part of $\Pi_{\mu \nu}$ for small coupling-i.e. the $g^{2} \log g$ terms - can be determined with no knowledge of $\Pi_{\mu \nu}^{\text {soft }}$, and using virtually none of the complications of the resummation formalism in $\Pi_{\mu \nu}^{\text {hard }}$.

The principal observation is that all of the terms in $\Pi_{\mu \nu}$ that are proportional to 
$g^{2} \log g$ are completely determined by the infrared divergent part of $\Pi_{\mu \nu}^{\text {hard }}$. To see how this works consider the lowest order contribution to the dispersion relation, $E(\mathbf{p})$, for transverse gluons with momenta $|\mathbf{p}| \sim T \gg g T$. This is determined from eq. (3) given the vacuum polarization, $\Pi_{\mu \nu}(E, \mathbf{p})$ evaluated at hard, on-shell momenta: $E=|\mathbf{p}| \sim T$. As is established in more detail below, the contribution of $\Pi_{\mu \nu}^{\text {hard }}$ to the right-hand-side of eq. (3) diverges logarithmically with $\lambda$ for $g^{2} T \ll \lambda \ll g T$ :

$$
\begin{aligned}
F^{\text {hard }}(T,|\mathbf{p}|, \lambda) & \equiv \frac{1}{2}\left[\left(\Pi^{\text {hard }}\right)_{i}^{i}-\frac{p^{i} p^{j} \Pi_{i j}^{\text {hard }}}{\mathbf{p}^{2}}\right] \\
& =g^{2}\left[A \log \left(\frac{\lambda}{\mu_{\text {hard }}}\right)+B+O\left(\frac{\lambda}{T}\right)\right]+O\left(g^{3}\right) .
\end{aligned}
$$

In this expression $A$ and $B$ are purely functions of $|\mathbf{p}|$ and $T$ and $\mu_{\text {hard }}$ is a calculable energy scale of the high-frequency part of the theory which turns out below to be $\mu_{\text {hard }} \approx g T$.

In order to extract information about $F(T,|\mathbf{p}|) \equiv F^{\text {hard }}(T,|\mathbf{p}|, \lambda)+F^{\text {soft }}(T,|\mathbf{p}|, \lambda)$ from eq. (6) it is necessary to say something about the behaviour of $F^{\text {soft }}(T,|\mathbf{p}|, \lambda)$. The only property of $F^{\text {soft }}(T,|\mathbf{p}|, \lambda)$ that is required is that its $\lambda$-dependence must cancel that of $F^{\text {hard }}(T,|\mathbf{p}|, \lambda)$ :

$$
\begin{aligned}
\lambda \frac{\partial F^{\mathrm{soft}}}{\partial \lambda} & \equiv-\lambda \frac{\partial F^{\mathrm{hard}}}{\partial \lambda} \\
& =g^{2}\left[-A+O\left(\frac{\lambda}{T}\right)\right]+O\left(g^{3}\right) .
\end{aligned}
$$

This determines $F^{\text {soft }}$ to have the form:

$$
F^{\mathrm{soft}}=g^{2}\left[A \log \left(\frac{\mu_{\mathrm{soft}}}{\lambda}\right)+C+O\left(\frac{\lambda}{T}\right)\right]+O\left(g^{3}\right),
$$

in which $A$ is the same function as in eq. (6). The constant $\mu_{\text {soft }}$ that appears within the logarithm in this equation is the constant of integration that arises in passing from eq. (7) to eq. (8). It has dimensions of mass and is chosen to be of order $g^{2} T$ since this is the largest mass scale present in the soft part of the problem.

Adding the results of eqs. (6) and (8) therefore gives:

$$
\begin{aligned}
F & =-A g^{2} \log \left(\frac{\mu_{\text {hard }}}{\mu_{\text {soft }}}\right)+g^{2}(B+C)+O\left(\frac{\lambda}{T}\right)+O\left(g^{3}\right) \\
& =-A g^{2} \log \left(\frac{1}{g}\right)+O\left(g^{2}\right),
\end{aligned}
$$


which determines the coefficient of the $g^{2} \log g$ term completely in terms of the calculable coefficient $A$.

The next point is that since $A$ is determined by the infrared divergent part of $F^{\text {hard }}$, it is insensitive to most of the complications of the resummation. To illustrate the simplicity with which $A$ may be determined we now outline its calculation for the damping constant.

Inspection of the Feynman rules shows that the only potentially infrared-divergent part of the leading contribution to $F^{\text {hard }}$ comes purely from the vacuum-polarization graph for $\Pi_{\mu \nu}^{\text {hard }}$ in which it is a gluon which circulates around the loop. Furthermore, even for this graph an infrared divergence can arise only from the term for which the integer $n=q^{0} /(2 \pi T)$ for the soft gluon line in eq. (5) vanishes, and even then only if the external four-momentum is on shell: $E=|\mathbf{p}|$. Since only $n=0$ contributes, it is sufficient to know the form for the resummed propagator at zero frequency, where it reduces to: ${ }^{5}$

$$
\left[\left(G^{*}\right)^{-1}\right]_{\mu \nu}(p)=\left(G_{\text {bare }}^{-1}\right)_{\mu \nu}(p)+m^{2} P_{\mu \nu}
$$

Here $P_{\mu \nu}$ is the projection matrix onto the rest frame of the plasma, and so is given in this frame by the matrix $\operatorname{diag}(0,1,1,1) . m$ denotes the lowest order gluon mass, or plasma frequency, which is given in terms of the number of quarks, $n_{q}$, by $m^{2}=\frac{1}{9}(g T)^{2}\left(C_{a}+\frac{n_{q}}{2}\right)$.

Substituting this into eq. (5), evaluating on the lowest-order mass shell, $E=|\mathbf{p}| \approx T$, and recognizing that at most one internal line can be soft at a time (and so need be dressed) then gives an infrared-divergent contribution:

$$
\begin{aligned}
\left.F^{\text {hard }}(T,|\mathbf{p}|)\right|_{\text {div }} & =-\frac{g^{2} C_{a} T m^{2}|\mathbf{p}|}{2 \pi^{2}} \int_{\lambda}^{\infty} d q \frac{1}{q\left(q^{2}+m^{2}\right)} \log \left(\frac{q-2|\mathbf{p}|+i \epsilon}{q+2|\mathbf{p}|}\right) \\
& =+\frac{i g^{2} C_{a} T|\mathbf{p}|}{2 \pi} \log \left(\frac{\lambda}{m}\right)
\end{aligned}
$$

from which we read $A=\left(i C_{a} T|\mathbf{p}|\right) /(2 \pi)$ and $\mu_{\text {soft }}=m$. Notice that the real part of $F$ is infrared finite so only the lifetime acquires a $g^{2} \log g$ contribution. Using this result in the mass-shell condition gives our main result:

$$
\begin{aligned}
\gamma_{g}(\mathbf{p}) & =-\frac{\operatorname{Im} F}{2|\mathbf{p}|} \\
& =+\frac{g^{2} C_{a} T}{4 \pi} \log \left(\frac{1}{g}\right)+O\left(g^{2}\right) .
\end{aligned}
$$


This entire argument when repeated for the quark self-energy similarly gives a $g^{2} \log g$ contribution to the branch of the fermion spectrum that survives at large momenta. A simple calculation gives its coefficient as $\gamma_{q}(\mathbf{p})=\frac{g^{2} C_{f} T}{4 \pi} \log \left(\frac{1}{g}\right)+O\left(g^{2}\right) . C_{f}$ denotes the quadratic invariant in the fundamental representation: $C_{f}=\left(N_{c}^{2}-1\right) /\left(2 N_{c}\right)\left(=\frac{4}{3}\right)$.

There are several features of this calculation that bear emphasis: (i) First, as is required for a good approximation to a physical quantity, $\gamma$ is independent of the gauge parameter $\xi$. All terms that depend on the gauge-parameter contribute only an infrared-finite result. (ii) The sign of $\gamma$ is positive, indicating stability. (iii) Notice that a determination of the subleading $O\left(g^{2}\right)$ contributions would require knowledge of both of the coefficients $B$ of eq. (6) and $C$ of eq. (8). Although $B$ is calculable using the complete resummation formulation, $C$ is not and can only at present be determined by making some assumptions concerning the behaviour of the plasma in the low-frequency regime $\lambda \approx g^{2} T$. It follows that the coefficient $B$ need not a priori by itself be gauge-independent (or positive). (iv) Also, since it is a logarithmic infrared-divergence that is responsible for the logarithmic dependence on $g$, its coefficient is insensitive to the details of how the cutoff is implemented. Finally, $(v)$ since the infrared-divergent term in $F^{\text {hard }}$ is explicitly proportional to $m^{2}$ (c.f. eq. (11)) it only receives contributions from the two-loop and higher graphs that serve to dress the soft propagator in the gluon vacuum-polarization graph. It also follows that the imaginary part arises only from the self-energy of internal lines which carry soft loop momenta $|\mathbf{q}|<g T$ since the mass $m$ may be taken to be zero for larger momenta. This agrees with what is expected physically from unitarity given the constraints of energy and momentum conservation in the plasma.

We conclude that for some quantities in which infrared divergences in the perturbative expansion introduce a logarithmic dependence on the gauge coupling, $g$, it is possible to very simply identify the dominant contributions. This simplicity allows a check on more complete and more involved calculations.

ACKNOWLEDGEMENTS: The authors would like to acknowledge helpful conversations with Charles Gale, Joe Kapusta, Randy Kobes, Gabor Kunstatter and Rob Pisarski, as well as funding by the Natural Sciences and Engineering Research Council of Canada and les Fonds pour la Formation de Chercheurs et l'Aide à la Recherche du Québec. 


\section{REFERENCES}

[1] O.K. Kalashnikov and V.V. Klimov, Sov. J. Nucl. Phys. 31 (1980) 699; D.J. Gross, R.D. Pisarski and L.G. Yaffe, Rev. Mod. Phys. 53 (1981) 43; U. Heinz, K. Kajantie and T. Toimela, Phys. Lett. 183B (1987) 96, Ann. Phys. (NY) 176 (1987) 218; K. Kajantie and J. Kapusta, Ann. Phys. (NY) 160 (1985) 477; J.C. Parikh, P.J. Siemens and J.A. Lopez, unpublished; T.H. Hansson and I. Zahed, Phys. Rev. Lett. 58 (1987) 2397, Nucl. Phys. B292 (1987) 725; H.-Th. Elze, U. Heinz, K. Kajantie and T. Toimela, Zeit. Phys. C37 (1988) 305; H.-Th. Elze, K. Kajantie and T. Toimela, Zeit. Phys. C37 (1988) 601; R. Kobes and G. Kunstatter, Phys. Rev. Lett. 61 (1988) 392; S. Nadkarni, Phys. Rev. Lett. 61 (1988) 396; M.E. Carrington, T.H. Hansson, H. Yamagishi and I. Zahed, Ann. Phys. (NY) 190 (1989) 373; J. Milana, Phys. Rev. D39 (1989) 2419; G. Gatoff and J. Kapusta, Phys. Rev. D41 (1990) 611; J. Kapusta and T. Tomeila, Phys. Rev. D39 (1989) 3197; R. Kobes, G. Kunstatter and K.W. Mak, Phys. Lett. 223B (1989) 433; S. Catani and E. d'Emilio, Phys. Lett. 238B (1990) 373, Nucl. Phys. B (19) ; K.A. James, Nucl. Phys. B (19) .

[2] R.D. Pisarski, Phys. Rev. Lett. 63 (1989) 1129, Nucl. Phys. A525 (1991) 175c; E. Braaten and R.D. Pisarski, Phys. Rev. Lett. 64 (1990) 1338.

[3] S. Weinberg, in the proceedings of the International School of Subnuclear Physics, Ettore Majorana, Erice, Sicily, Jul 23 - Aug 8, 1976 (QCD161:I65:1976).

[4] For a discussion see, for example, J. Kapusta, Finite Temperature Field Theory, (Cambridge University Press, 1985).

[5] E. Braaten and R.D. Pisarski, Phys. Rev. Lett. 64 (1990) 1338, Nucl. Phys. B337 (1990) 569.

[6] V.V. Lebedev and A.V. Smilga, University of Berne preprints BUTP-89/25 and BUTP-90/38 (unpublished).

[7] R.D. Pisarski, Phys. Rev. Lett. 63 (1989) 1129; and E. Braaten and R.D. Pisarski, unpublished.

[8] E. Braaten and R.D. Pisarski, unpublished.

[9] T. Matsubara, Prog. Theor. Phys. 14 (1955) 351. 